\title{
THE SURGICAL ANATOMY OF RAMMSTEDT'S OPERATION
}

BY

DENIS BROWNE, F.R.C.S.,

Surgeon to the Hospital for Sick Children, Great Ormond Street, London.

It is generally admitted that Rammstedt's operation is not one of the easier ones of surgery, quite apart from the smallness and weakness of the patient. The main reason for its difficulty is that, while there is no margin whatever between the disaster of doing too little and the danger of doing too much, there are no clear indications to show that the object of the operation has been accomplished. In the published accounts there is a lack of definite landmarks to guide the inexperienced in the division of the muscle, and certain curious points about the relations of the different structures have never, as far as I know, been described. I have found the recognition of two of these, the narrowing of the bore of the mucosa at the pyloric orifice, and the zone of adhesion that follows it, of very considerable help in the operation.

The structures encountered in the freeing of the pylorus fall into five main groups. I propose first to describe them shortly, and then to show how their relations affect the operation and the various mischances that may occur in the course of it.

1. The serous coat.-This has no particular features to distinguish it from the visceral peritoneum covering the alimentary tract elsewhere. Two of its qualities which are of importance in the operation are that it is extremely adherent to the muscle beneath, and that it is tough and inextensible.

2. The blood vessels.-These run from two sources, the right gastric and gastro-epiploic vessels, and in two planes, a superficial and a deep. The superficial ones show conspicuously on the surface just under the serosa, but in the region of the operation they fail to make any visible anastomosis at the mid-point from their origins, as they do elsewhere on the stomach. The hiatus between them forms the well-known 'bloodless line' which follows the axis of the viscus, and so is curved upwards at its left extremity.

The deeper vessels mostly ramify in the mucosa, but there are two which are almost invariably seen in the muscle during the operation. The most important of these is an artery, with its accompanying vein, which is met running across the duodenal end of the incision, just at the apex of the fornix of the mucosa, to which it forms a very useful indication. The other vessel appears in the deeper layers of muscle towards the other end of the wound, and occasionally causes trouble from bleeding.

3. The muscular coat.-The only known pathological change in the discase is an cver-growth of muscular fibres, especially at the pylorus, and this results in a blockage there that is mechanical rather than functional ${ }^{1}$.

This swelling of the muscle takes place inside an adherent and unstretchable covering of peritoneum, and consequently can go in two directions only, 
inwards into the lumen, and lengthways, mainly into the duodenum. The result of the swelling inwards is that the pylorus, instead of being when relaxed the normal limp and patent tube, remains at all times a hard and solid mass with the muscle tightly filling the serous coat, as a cork does the neck of a bottle. The swelling downwards produces a protrusion of the pyloric orifice into the duodenum which is usually compared to that of the os uteri into the vagina, with, of course, a similar fornix of mucous membrane surrounding it.

Another mechanical effect of the confinement and hypertrophy of the muscle is a decrease in its vascularity, from the compression of the vessels supplying its fibres. At operation it is noticeable that there is much less oozing from the cut surfaces of the hypertrophied sphincter than there is from the normal fibres that may be cut at either end of it, or than there is if a normal pylorus be incised.

Finally the muscle is changed in consistency from its usual fibrous state to a crispness resembling that of young celery. As with celery, toughness ensues with age, and in the later stages of the disease the muscle is noticeably more fibrous and vascular than in the early ones. Presumably this is due to the beginning of that return to normal which will become complete if the child survives.

4. The areolar or submucous coat.-This inconspicuous layer makes the operation possible, and to understand its relations it is necessary to study its functions. Here as elsewhere in the body the areolar tissue exists to form a connection that will allow two structures to move freely on one another, and, as usual, one of these structures is muscle. The other is the mucosa, and the need for independent movement arises because it, being non-contractile, must meet the contractions of the surrounding muscle by wrinkling into folds inside it.

As might be expected, the amount and looseness of the areolar tissue and the depths of the folds are greatest at the points where the variations in diameter of the tube are greatest, and least where they are least. Thus the areolar layer is well marked in the pyloric antrum, becomes still wider and looser up the pyloric canal, decreases markedly over the projection of the sphincter, ceases entirely at the apex of the fornix, and then reappears again in the duodenum.

5. The mucosa.-This is the most important of all the layers, and the most difficult both to understand and to describe. In the pyloric antrum it is of considerable thickness and is wrinkled into longitudinal folds, which increase in depth as the pyloric canal is reached, and so give the well-known star shape of the potential cavity that is seen here on transverse section. These deep folds run through the canal till the actual opening into the duodenum is reached, when they suddenly cease just as the bore of the muscular tube starts to widen. Consequently at the apex of the pyloric protrusion into the duodenum, although the actual diameter of the mucous tube is far greater than that in the pyloric canal, the potential diameter is far less. In other words, if the entire mucosa in this region be freed of muscle and dilated to its full extent, it will be found that at the pyloric orifice there is a very sudden 
and well marked 'choke' in its bore, like that at the muzzle of some shotgun barrels.

At the end of this zone of constriction, which only lasts for about an eighth of an inch, the mucous membrane suddenly expands into the full diameter of the duodenum, which is approximately twice that of the choke and the same as that of the potential diameter of the pyloric portion. It runs backwards smoothly applied to the protruding nozzle. of muscle to form the fornix already mentioned, and then forward again as the lining of the duodenum.

On consideration it will be seen that the apex of this fornix is subject to a considerable force tending to tear it downwards into the duodenum whenever

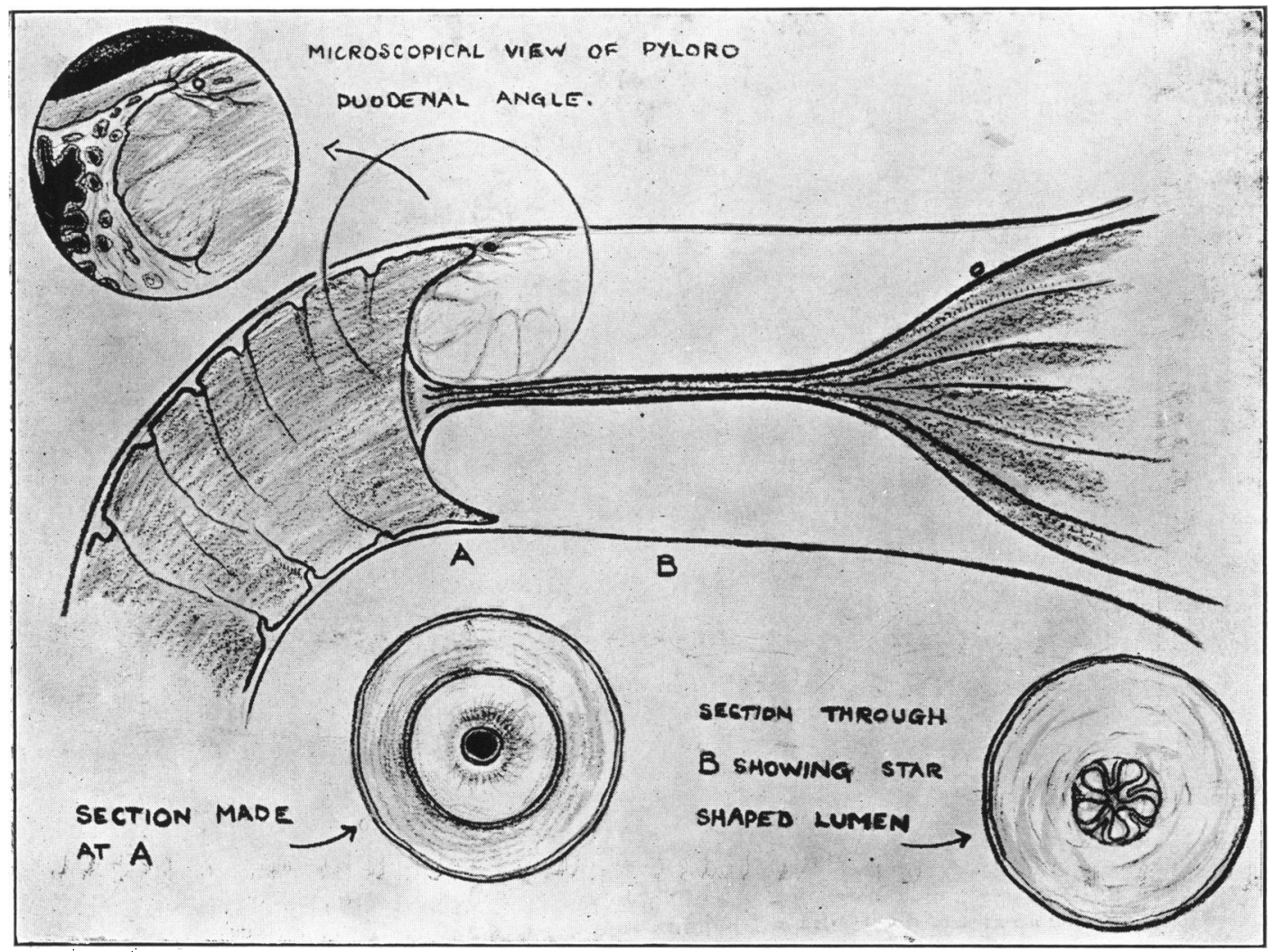

Frg. 1. Semi-diagrammatic sections of the hypertrophied pylorus. The microscopical view shows the fibrous bands radiating from the fornix of the mucosa, and the vessel running just outside it.

the stomach contracts against the stopper-]ike mass of muscle in the pylorus ; and as might be expected, this force is counteracted by a development of fibrous tissue, as in the similar case of the tonsil ${ }^{2}$. The fibres run, just as Lane's doctrine of the organization of lines of force would lead one to expect, outwards and backwards from the angle of reflection of the mucosa, into the muscle. In consequence a ' zone of adhesion' is formed at this point, in which mucosa, muscle, and serosa are bound into one almost inseparable mass, but immediately beyond it the mucosa once again becomes rugose and lies loosely attached to the duodenal muscle (Fig. 1). In this area the mucous membrane 
is a very vascular and vital structure, which can be stripped of all protection over a large area without any risk of sloughing. It is also, like all sheets of tissue, of surprising tensile strength as long as it remains uninjured, but once it has been punctured the tear will extend with the greatest readiness.

A final point for consideration is that the folds in the pyloric antrum may possibly form valves over the opening of the canal if this is allowed to remain very small. Whether this ever occurs in life I am not prepared to say, but it is certainly possible after death to produce a valve of this sort at the other end of some stomachs. In these water may be run in through a tube

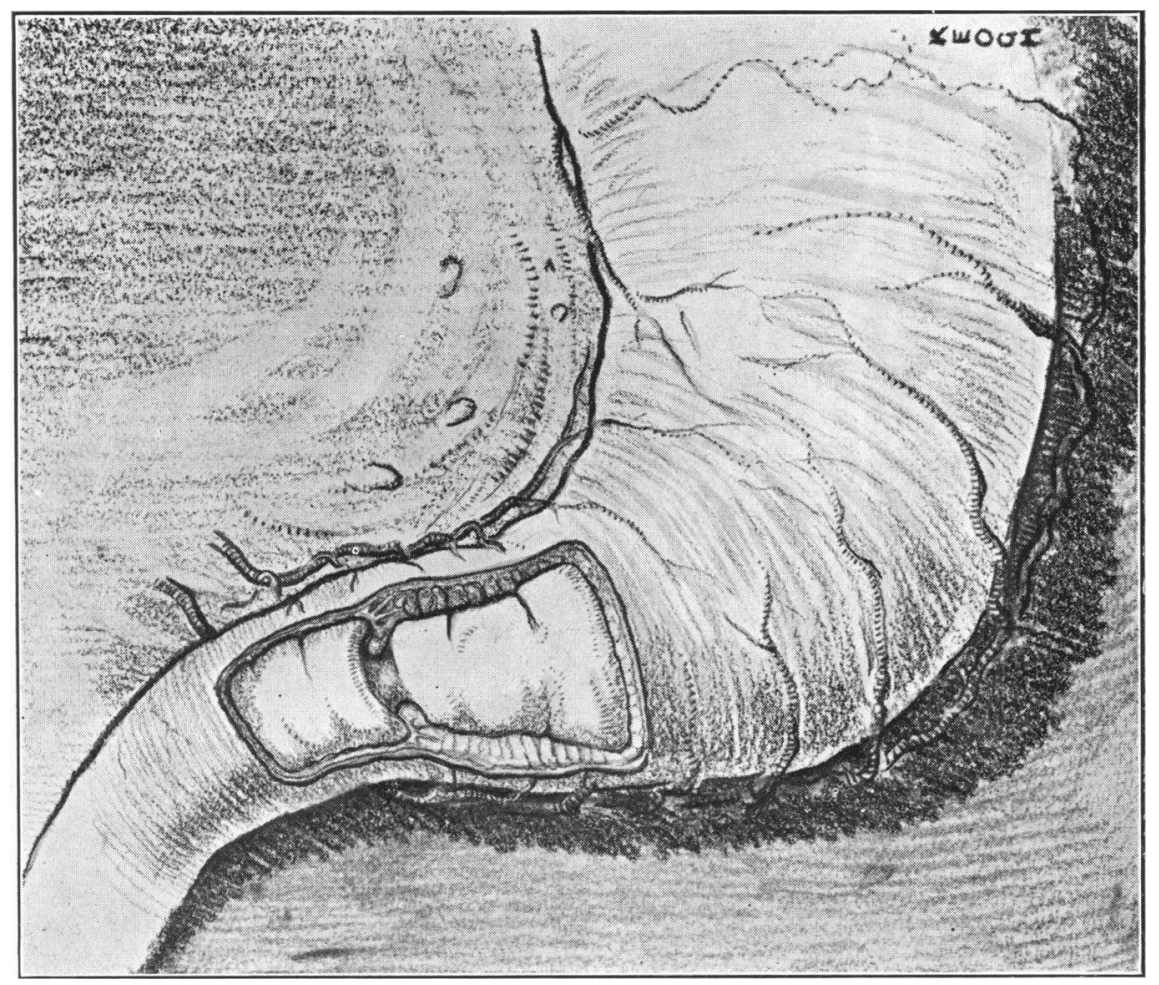

Fic. 2. Dissection of stomach of a case of hypertrophic stenosis with the muscle removed from an area of duodenum and pylorus, showing zone of constriction of mucosa.

in the cardiac orifice till it is under considerable pressure, but on withdrawing the tube folds of mucous membrane fall together to produce a valve and prevent its escape.

To confirm these observations it is necessary to make a rather difficult dissection. The first step in it is to fill the stomach with water to a fairly high tension by means of a tube passed into the cardiac orifice, after the duodenum has been closed by a ligature. Then an oblong area, as shown in Fig. 2, is marked out by an incision through the serous and muscular coats, and these are peeled off the mucosa from either end in towards the junction of the pylorus and duodenum. It will be found moderately easy to do this 
till the adherent zone is reached; but here, as the tensile strength of the mucosa is less than that of its adhesion to the muscle, a separation can only be made by clean cutting. The best way at this point is to leave this patch of muscle adherent till the preparation has been hardened and fixed in its expanded state. It can then easily be cut away, and the result is as shown in Fig. 2, with the bulging mucosa clearly showing the zone of constriction.

\section{Discussion.}

The practical application of these points is fairly obvious. The right end of the incision is determined by the always clearly visible junction of the

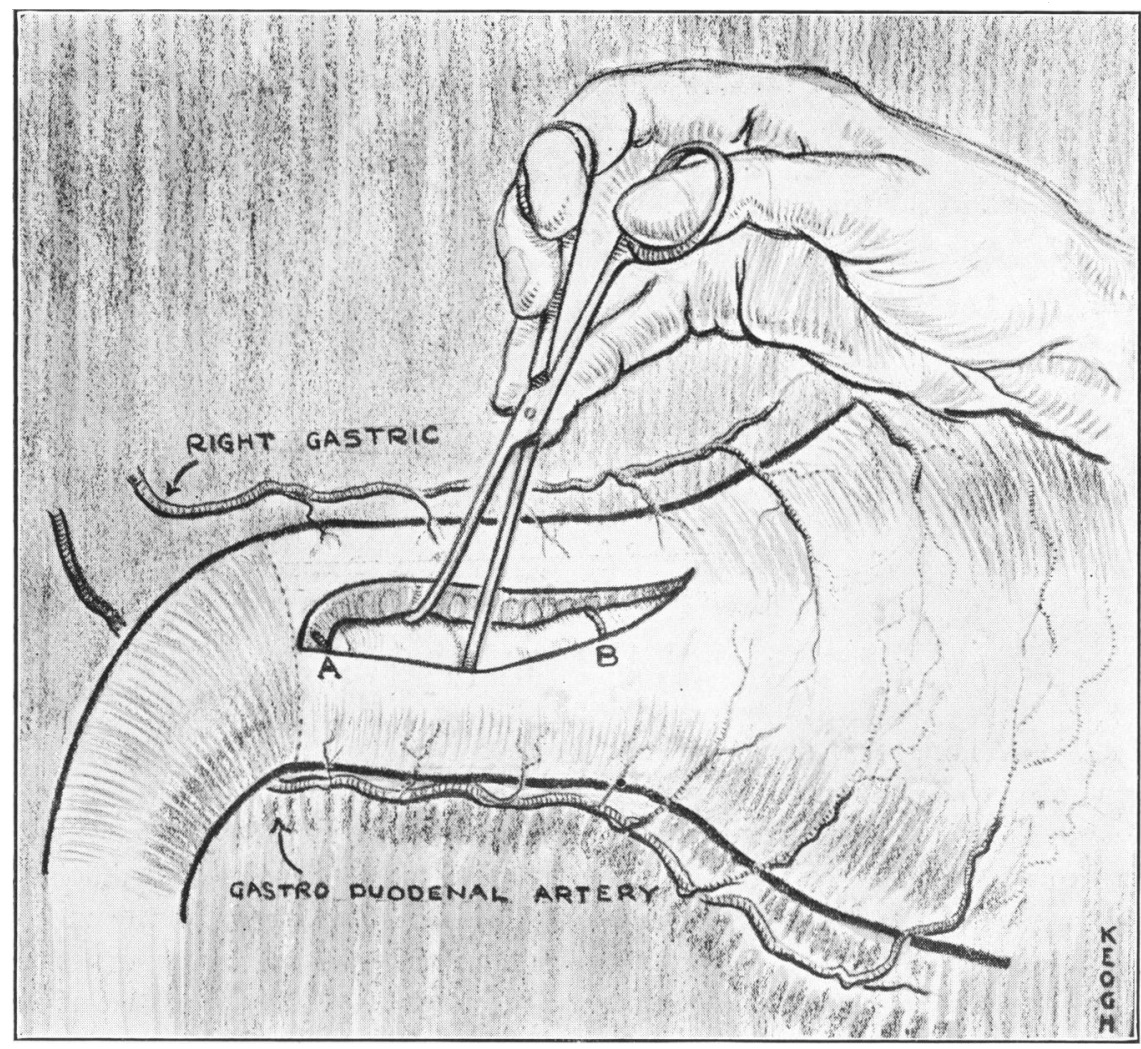

Fig. 3. Semi-diagrammatic drawing of Rammstedt's operation showing the vessels at either end of the incision, and the narrowing of the bore of the released mucosa at the zone of constriction.

whitish, opaque, and solid pylorus with the bluish, translucent, and flaccid duodenum. The cut should begin a millimetre or two short of this, and extend well into the pyloric antrum, curving upwards in its left third to follow the bloodless line between the superficial blood vessels. Most illustrations give the impression of too short an incision, and as there is no definite anatomical limitation in this direction, there is no reason for risking possible blockage here by curtailing the cut. 
The muccsa is then exposed by blunt dissection, but exposure is far from being enough. The aim of the operation is not so much to allow the passage of food through the original pyloric canal, as to form a totally new one alongside it, by the bulging out of the uncovered mucosa. To do this at least half an inch of it should be exposed in the middle of the incision and as near as possible to either end. By far the easiest and safest way to do this is by stretching the edges of the incision in the muscle apart, so that they tear away from the intact mucosa beneath. Owing to the looseness of the subjacent areolar tissue this is perfectly safe and easy to do over the pylorus and on to the stomach, but it is a different matter at the duodenal end. Here the zone of adhesion is the danger, as although the muscle will split from the mucosa as easily in the duodenum as in the stomach, there lies between these two regions this ring in which serosa, muscle, and mucosa are all bound together, and will split together or not at all. If it were not for this it would be possible to divulse the muscle just as boldly at this end of the wound as at the other, and to leave the mucosa to take care of itself.

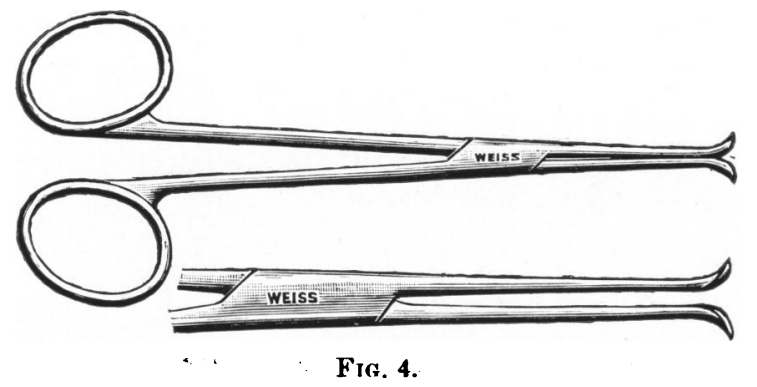

In avoiding this dangerous region there are two aids. The first is to remember that it is marked by the vessel described, and lies superficially, so that while it is risky to extend the wound near the surface, it is comparatively safe to stretch the deep muscle fibres till they part. The second aid is the recognition of the annular constriction, which is announced by the mucous membrance suddenly ceasing to bulge when it is released, and dipping down in the choke described. Thus the sign that the obstruction has been overcome is the paradoxical one that the calibre of the released mucosa suddenly narrows.

For the purpose of stretching the sides of the muscular incision apart no instrument in the surgeon's usual kit is really suitable, and I think it so important to have a complete control of this manœuvre that $I$ have got Messrs. Weiss to make me a small stretcher with carefully blunted ends set at the correct angle to bite in the tough walls (Fig. 4).

If the surgeon is in any doubt about the freeing of a passage, he can test the duodenal end of it by invaginating the wall of the duodenum with a blunt pointed pair of artery forceps, and pushing them through the pyloric opening till they can be felt under the freed mucosa. This is usually quite easy to do, but in some cases the pylorus cannot be delivered far enough to allow it. 
Mischances of the operation.-The first of these is the opening of the abdomen on a wrong diagnosis, because of spasm of the pylorus instead of true hypertrophic blockage. I have done this twice, and on each occasion cured the vomiting by performing Rammstedt's operation on the normal pylorus, throwing it out of action just as the sphincter ani is put at rest by division in treating a fissure. I had learnt by post-mortem experiment that it is easy enough to divide the normal pyloric muscle, and though no one would deliberately operate for pylorospasm I see no contra-indication to cutting the sphincter once the pylorus is exposed.

The second mischance is the puncturing of the mucosa, and though this is alarming enough, it is less serious than might be expected. I know of at least six cases in which it has happened, but none of them resulted fatally. The reason for this may be that the point at which it invariably occurs, the end of the fornix of the mucosa, on the upper side, is almost entirely immune from the pressure, or even the contact, of fluid passing down the duodenum.

The third and most serious of the mischances is the failure to relieve the blockage of the pylorus, and when this occurs the only chance lies in a second operation to complete what should have been done at the first. I have had one case of this sort, and though the child ultimately recovered, it was that contretemps which stimulated me to the studies resulting in this paper.

I have to thank Dr. C. A. Keogh for his excellent drawings, and also for suggesting the real function of the zone of adhesion.

\section{REFERENCES.}

1. Thompson, .J. .J., Brit. Merl. .J., London, 1921, ii, 889.

2. Browne, D., J. Anat., London, 1928, LXIII, 82.

3. Downes, W. A., J. Amer. Med. Ass., Chicago, 1920, LXXV, 228.

4. Gray, H. T., Brit. Med. J., London, 1921, ii, 891. 